and non-ILD and b) limited vs. extensive ILD patients. Spearman rank correlation was performed to reveal significant associations of radiomic features from a) and $b$ ) with lung function as measured by percentage of predicted forced vital capacity (FVC\% predicted).

Results: In total, $1355 / 1386$ radiomic features passed the test of robustness and were eligible for further, exploratory analyses. Radiomic features with good performance (area under the ROC curve (AUC) $\geq 0.7$ and $p$-value $\leq 0.05$ ) were considered as potential candidate discriminators. Under this criterion, we identified $288 / 1355(21.3 \%)$ radiomic features that were significantly different between ILD and non-ILD patients and 409/1355 (30.2\%) features that significantly discriminated between limited and extensive ILD (Fig. 1). For diagnosis, the texture feature dependence count entropy was the top parameter to distinguish ILD patients from healthy controls $\left(A \cup C=0.89, p=1.83 \times 10^{-10}\right.$ ), whereas for staging the wavelet feature $\mathrm{HHH}$ long run high grey level emphasis proved to be best suited to separate limited from extensive ILD ( $\left.A U C=0.88, p=7.76 \times 10^{-9}\right)$.
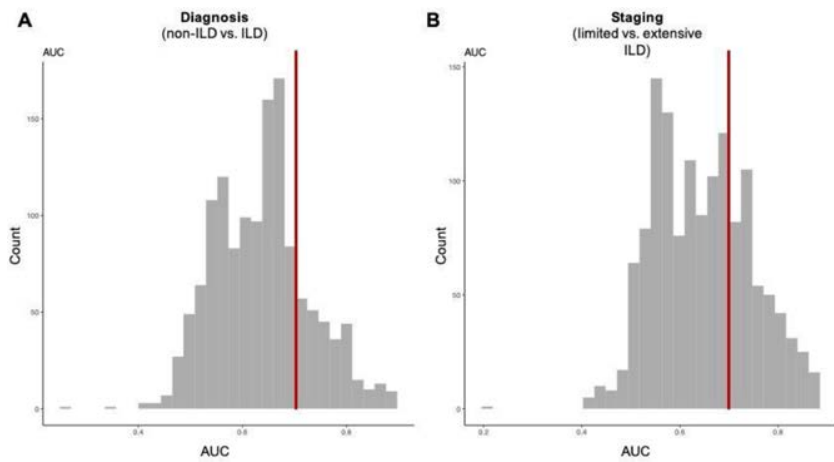

Fig 1.

Correlation analysis of the most significant (best performing) discriminative radiomic features with lung function revealed a significant negative correlation of dependence count entropy (rho $=-0.51, \mathrm{p}=9.89 \times 10^{-8}$ ) and $\mathrm{HHH}$ long run high grey level emphasis ( $r$ o $=-0.51, \mathrm{p}=1.73 \times 10^{-5}$ ) with $\mathrm{FVC} \%$ predicted.

Conclusion: Our study adds novelty to the field of SSc-ILD showing that radiomic features have great potential as quantitative imaging biomarkers for diagnosis and staging of SSc-ILD and that they may reflect lung function. As the next step, we are planning to build predictive models, using machine learning, for diagnosis, staging, and lung function and validate them in external patient cohorts. If validated such models will pave the way for computer-aided management in SSc-ILD and thus improve patients' outcome.

References:

[1] Gillies, R. J., Kinahan, P. E. \& Hricak, H. Radiomics: Images Are More than Pictures, They Are Data. Radiology 278, 563-577, doi:10.1148/ radiol.2015151169 (2016).

Disclosure of Interests: Janine Schniering: None declared, Malgorzata Maciukiewicz: None declared, Hubert Gabrys: None declared, Matthias Brunner: None declared, Christian Blüthgen: None declared, Oliver Distler Grant/research support from: Grants/Research support from Actelion, Bayer, Boehringer Ingelheim, Competitive Drug Development International Ltd. and Mitsubishi Tanabe; he also holds the issued Patent on mir-29 for the treatment of systemic sclerosis (US8247389, EP2331143)., Consultant of: Consultancy fees from Actelion, Acceleron Pharma, AnaMar, Bayer, Baecon Discovery, Blade Therapeutics, Boehringer, CSL Behring, Catenion, ChemomAb, Curzion Pharmaceuticals, Ergonex, Galapagos NV, GSK, Glenmark Pharmaceuticals, Inventiva, Italfarmaco, iQvia, medac, Medscape, Mitsubishi Tanabe Pharma, MSD, Roche, Sanofi and UCB, Speakers bureau: Speaker fees from Actelion, Bayer, Boehringer Ingelheim, Medscape, Pfizer and Roche, Matthias Guckenberger: None declared, Thomas Frauenfelder: None declared, Stephanie Tanadini-Lang: None declared, Britta Maurer Grant/ research support from: AbbVie, Protagen, Novartis, congress support from Pfizer, Roche, Actelion, and MSD, Speakers bureau: Novartis DOI: 10.1136/annrheumdis-2020-eular.928

\section{SAT0570 CLINICAL SIGNIFICANCE OF FINGER EXTENSOR PARATENONITIS DETECTED BY MUSCULOSKELETAL ULTRASOUND}

T. Suzuki ${ }^{1}$, H. Shirai ${ }^{1}$. Japanese Red Cross Medical Center, Division of Allergy and Rheumatology, Tokyo, Japan

Background: The extensor tendons over fingers are devoid of a tendon sheath, so that the term paratenonitis is used to describe extra-articular hyperemia or anechoic fluid collections along the extensor tendons of the fingers. Although the grading of paratenonitis is found in one sonographic scoring system of RA known as German US7, the clinical significance of paratenonitis is not fully understood. Objectives: To determine the clinical significance of finger extensor paratenonitis detected by ultrasound (US), especially in the patients with RA

Methods: We reviewed 1200 reports of the US examination underwent in ou division since April 2015. The items necessary for scoring US5 scores (the 'hand-limited version' of the German US7) have been routinely recorded. The cases with finger extensor paratenonitis over the dorsal metacarpophalangeal joint (MCPJ) were determined. The severity of articular synovitis in the perilesional MCPJ were subjectively graded for grey-scale (GS) and power Doppler (PD) on a four-step scale (0-3) and scored using EULAR-OMERACT combined scoring system. In RA patients, US5 scores were determined for the involved hands.

Results: Paratenonitis was found in 44 fingers in the 38 hands of the 36 patients with rheumatic diseases/disorders including 25 patients with RA (11 early RA and 14 established RA). Non-RA diseases/disorders included 4 cases of undifferentiated arthritis, 2 cases of PsA, 1 case each of SLE, Sjogren syndrome, reactive arthritis and other disorders.



The 44 fingers were classified according to the absence or presence of articular synovitis in the perilesional MCPJ into "isolated paratenonitis" or "paratenonitis accompanied by synovitis". The distribution of paratenonitis over the $1^{\text {st }}-5^{\text {th }}$ fingers of the dominant or non-dominant hands is shown in Figure 1. Paratenonitis was relatively frequently found in the $3^{\text {rd }}$ and $2^{\text {nd }}$ fingers of the dominant hands. Interestingly, articular synovitis in the perilesional MCPJ were found significantly more frequent in the cases of MCP2 in the dominant hands (73\%) than in the cases of MCP3 in the dominant hands $(25 \%)(p=0.039)$.

Figure 2
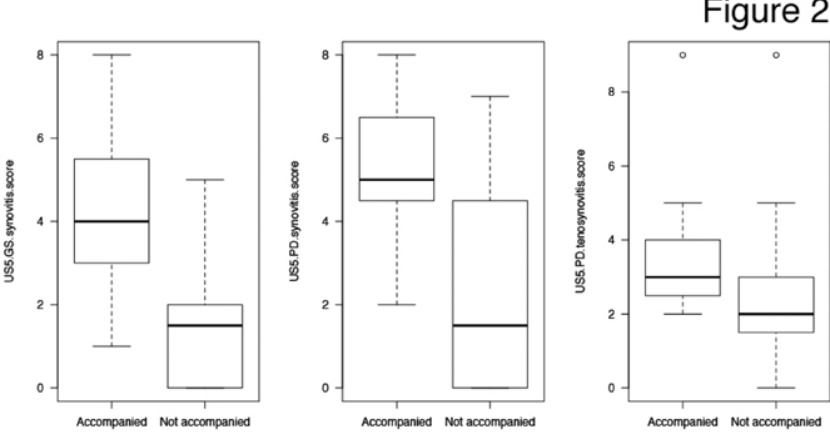

Hands with paratenonitis accompanied or not accompanied by moderate to severe MCPJ synovitis

Among the 27 hands with paratenonitis of 25 RA patients, US5 scores were compared based on the absence or presence of moderate to severe articular synovitis in the perilesional MCPJ (the combined score >1) (Figure 2). GS synovitis score, PD synovitis score and PD tenosynovitis score were significantly higher in those with moderate to severe perilesional MCP synovitis than in those without it ( $\mathrm{p}=0.007,0.0092$ and 0.0458 , respectively).

Conclusion: Finger extensor paratenonitis over the dorsal MCPJ tends to occur in the $3^{\text {rd }}$ and $2^{\text {nd }}$ fingers of the dominant hand. In RA patients, paratenonitis accompanied by active perilesional MCPJ synovitis are presumably due to active disease, while isolated paratenonitis can also be caused possibly by degenerative changes due to overuse or deformity. Isolated paratenonitis may be more frequently found in the $3^{\text {rd }}$ finger than in the $2^{\text {nd }}$ finger of the dominant hand. 
References: None

Disclosure of Interests: Takeshi Suzuki Speakers bureau: Abbvie, Asahi-kasei, Astellas, Bristol-Myers Squibb, Chugai, Eli Lilly, Mitsubishi-Tanabe, Novartis, UCB, Harumi Shirai: None declared

DOI: 10.1136/annrheumdis-2020-eular.1944

\section{SAT0571 OPTICAL SPECTRAL TRANSMISSION TO ASSESS THERAPY RESPONSE IN PATIENTS WITH ARTHRITIS: A COMPARATIVE STUDY WITH CLINICAL, LABORATORY AND ULTRASONOGRAPHIC ACTIVITY MARKERS.}

K. Triantafyllias ${ }^{1}$, C. Heller ${ }^{2}$, M. De Blasi ${ }^{1}$, M. Muthuraman ${ }^{3}$, A. Schwarting ${ }^{1,2}$. ${ }^{1}$ ACURA Clinics, Rheumatology, Bad Kreuznach, Germany; ${ }^{2} J o h a n n e s$ Gutenberg University Medical Center, Internal Medicine I, Deparmtnet of Rheumatology and Clinical Immunology, Mainz, Germany; ${ }^{3} J o h a n n e s$ Gutenberg University Medical Center, Biomedical Statistics and Multimodal Signal Processing, Mainz, Germany

Background: Valid assessment of disease activity leads to outcome improvement in patients with rheumatoid arthritis (RA) (1). Optical spectral transmission (OST) is a modern diagnostic tool able to assess the blood-specific absorption of light transmitted through a tissue, promising quantification of inflammation in the finger and wrist joints of RA patients (commercial device: HandScan - Hemics, The Netherlands) (2).

Objectives: To our knowledge, there are no data regarding the diagnostic value of OST in the evaluation of inflammatory activity changes during arthitis follow up. Thus, aims of this study were to examine the ability of OST to detect response to anti-inflammatory therapy in patients with arthritis and to explore OST associations with clinical, laboratory and ultrasonographic (US) activity markers.

Methods: OST measurements were performed in patients with active arthritides of the wrist and finger joints before and after administration of glucocorticoids (GC), during a disease flare. For the same points in time ( $a$ and $b$ ) patients and healthy controls underwent clinical, laboratory and joint US [Grey Scale (GSUS) Power Doppler (PDUS)] examinations. OST-values before and after therapy were subsequently compared with their corresponding DAS28- and US-values. The distributions of Delta-PDUS und OST-values between the two time points were compared by Bayesian statistics. Moreover, OST diagnostic performance was tested by Receiver Operating Characteristics (ROC).

Results: We recruited 54 patients with active inflammatory arthritis: 39 RA, 4 gout, 7 peripheral spondylarthritides and 4 other miscellaneous arthritides (66.7\% females) and 114 controls.

Previous to therapy with GC, median OST was [OST(a): 8.75 (5.38-16.25, IQR)] and after therapy [OST(b): $4.75(2.38-8.63, \mathrm{IQR})](p<0.05)$. Similarly, DAS28 dropped significantly after GC therapy [DAS28(a): 5.12 (4.33-6.10, IQR) vs. DAS28(b): 3.85 (3.40-4.82), $p<0.05)$ ]. OST correlated moderately with PDUS at both time points: (a) $r h o=0.449$ and (b) $r h o=0.414$, respectively (both; $p<0.01$ ). Moreover, OST correlated significantly with swollen joint count at both time points (a) $r h o=0.379$ and (b) $r h o=0.382, p<0.01$ respectively.

OST and US performed similarly in the assessment of inflammatory changes caused by the administration of GC (same tendency in the change of OST values in $83.2 \%$ of the cases). Furthermore, Bayesian statistic revealed no significant differences between OST and US for all 3 examined joint categories (MCP: $p=0.81$; PIP: $p=0.74$; wrists: $p=0.60$ ).

In addition, ROC revealed that OST is a very good tool to distinguish patients with arthritis from healthy controls at both examination points [AUC(a): 0.883(95\% $\mathrm{Cl}=0.83-0.94)$ and $\mathrm{AUC}(\mathrm{b}): 0.811(95 \% \mathrm{Cl}=0.74-0.881)]$.

Conclusion: OST was able to assess response to therapy in arthritis patients comparable to US. Moreover, OST correlated with disease activity markers and could effectively differentiate between arthritis patients and controls. Therefore, OST could prove to be a valuable non-interventional time- and resource-saving diagnostic tool to assist arthritis monitoring.

References:

[1] Katchamart W, et al. Systematic monitoring of disease activity using an outcome measure improves outcomes in rheumatoid arthritis. J Rheumatol 2010;37:1411-1415.

[2] Onna M Van, et al. Assessment of disease activity in patients with rheumatoid arthritis using optical spectral transmission measurements, a non-invasive imaging technique. Ann Rheum Dis 2016;75:511-518.

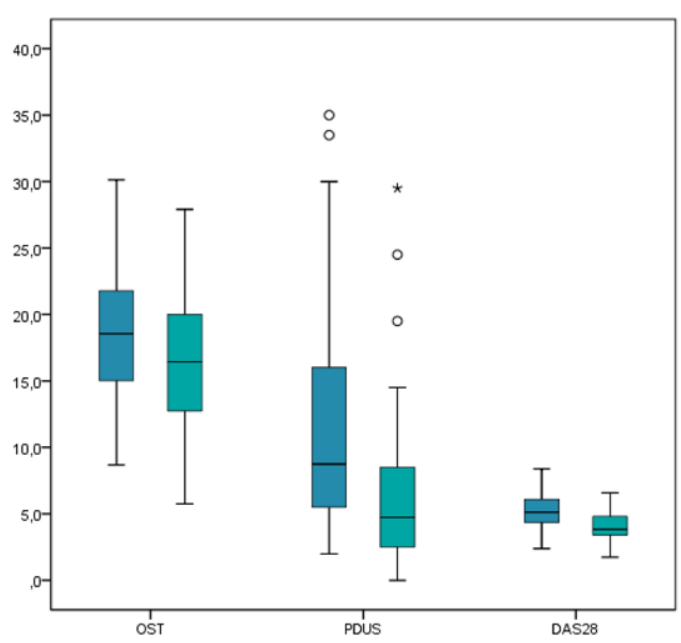

Figure 1. OST-, PDUS- and DAS28- values before and after GC therapy.


Figure 2. OST- (group 1) und Ultrasound- (group 2) Bayesian distributions, means- and standard deviation-differences for MCP (A), PIP (B) and wrists (C).

Disclosure of Interests: None declared

DOI: 10.1136/annrheumdis-2020-eular.3028

\section{SAT0572 CONSTRUCTIVE VALIDITY OF MUSKULOSKELETAL ULTRASOUND MEASUREMENT OF CARTILAGE THICKNESS IN PATIENTS WITH KNEE OSTEOARTHRITIS}

Z. Velickovic ${ }^{1}$, S. Janjic ${ }^{1}$, V. Bajec ${ }^{1}$, B. Stojic ${ }^{1}$, T. Zivanovic Radnic ${ }^{1,2}$, M. Rasic ${ }^{1}$, G. Radunovic ${ }^{1,2} .{ }^{1}$ Institute of Rheumatology, Belgrade, Serbia; ${ }^{2}$ School of Medicine, University of Belgrade, Belgrade, Serbia

Background: Cartilage thickness is one important measure in describing both OA development and progression. Based on current knowledge, conventional radiography $(\mathrm{CR})$ and magnetic resonance imaging (MRI) have not been demonstrated to be superior over one another. Because of disadvantages of MRI and $C R$ neither can be use in routine daily clinical practice for follow up of OA patients. Diagnostic ultrasound assessment (US) of cartilage thickness offers an alternative measure as a clinically available and more cost-effective source of knee articular cartilage imaging.

Objectives: Our objective was to determine the relationship between US and CR measures of femoral cartilage thickness in patients with knee osteoarthritis because systematic feature- and site-specific cross-comparison between this two methods is still missing in the current literature.

Methods: 120 patients with knee osteoarthritis (240 knees) are recruited for this study. The joint space width (JSW) and Kellgren and Lawrence (K\&L) grade were measured using weight-bearing anteroposterior $30^{\circ}$ knee semi-flexion knee radiography (with inclusion criteria K\&L grade 1-4). Femoral cartilage thickness was measured three times in supine position and with a suprapatellar transverse scan with the knee in maximal flexion at the lateral condyle (LC), medial condyle (MC) and intercondylar notch (IN) by one rheumatologist and arithmetic mean is taken. Pain and 